\title{
MEASURE PRESERVING HOMEOMORPHISMS AT FIXED POINTS
}

\section{DEANE MONTGOMERY}

In an article of a few years ago [2] ${ }^{1}$ Kerékjártó obtained interesting results about certain types of transformations which he called similitudes. With a few modifications and extensions his methods can be used to gain information about the structure of measure preserving transformations at fixed points. For simplicity the results are formulated for Euclidean $n$-space although they could easily be given a much more general setting and in particular the relevant ones apply to any $n$-dimensional manifold on which there is defined a measure satisfying light restrictions. Actually, as in most topological investigations of measure preserving transformations, the main property needed is that a bounded open set can not be carried into a subset of itself such that the difference of the two sets contains interior points.

It is shown that there are compact continua of assorted sizes which contain the fixed point and which are carried into themselves by the transformation. Such continua might, for example, be solid spheres $x_{1}^{2}+\cdots+x_{n}{ }^{2} \leqq r^{2}$ as in the case of an orthogonal transformation. On the other hand they might be arcs as in the case of the transformation

$$
x_{1}^{\prime}=2 x_{1}, \quad x_{n-1}^{\prime}=2 x_{n-1}, \quad x_{n}^{\prime}=1 / 2^{n-1} x_{n},
$$

where continua of the type described are intervals on the $x_{n}$-axis which include the origin.

The results also show that there are certain points near the fixed point which remain near it under indefinite positive iteration of the transformation. We use the symbol $U^{-1}$ for the set $T^{-1}(U)$, and so on.

THEOREM 1. Let $T$ be a measure preserving homeomorphism of $E_{n}$ onto itself, and let $A$ be a compact connected set such that $T(A) \subset A$. Then if $U$ is an open set with compact closure which includes $A$, there exists a compact connected set $K$ of which $A$ is a proper subset and such that $K$ is in $\bar{U}^{-1}$ and $T(K) \subset K$.

The theorem applies to the particular case where $A$ consists of a single fixed point. We divide the proof into two cases.

Case I. Assume that there exists an open connected set $V$ in $U^{-1}$

Presented to the Society, September 17, 1945; received by the editors August 20, 1945.

${ }^{1}$ Numbers in brackets refer to the Bibliography at the end of the paper. 
which includes $A$ and which is such that $T(V) \subset V$.

In this case $\bar{V}$ may be taken as the desired continuum because if either $T(V)$ is in $V$ or $T^{-1}(V)$ is in $V$, then $T(\bar{V})=\bar{V}$. In order to see this suppose for example that $T(V)$ is in $V$ so that we have immediately $T(\bar{V}) \subset \bar{V}$. If $T(\bar{V})$ did not include all of $\bar{V}$, then because of the nature of $\bar{V}$ it would have to omit some interior points of $\bar{V}$ and hence could not have the same measure as $\bar{V}$. When $T^{-1}(V)$ is in $V$, the procedure is similar and in either event we have $T(\bar{V})=\bar{V}$ which is even stronger than the stated conclusion.

Case II. Assume that there exists no open connected set $V$ in $U^{-1}$ with the properties described in case I. Both $U^{-1}$ and $U$ include $A$. Let

$$
U_{1}^{1}=C\left(U^{-1} \cap U\right)
$$

where $C\left(U^{-1} \cap U\right)$ is used to designate the component of $U^{-1} \cap U$ which includes $A$. We also have

$$
U_{1}=C\left(U^{-2} \cap U^{-1}\right),
$$

where, as usual, superscripts are used to indicate positive or negative iterates of a set, and subscripts merely distinguish between different sets. We see that $U_{1}$ is not contained in $U$, because $U_{1}$ in $U$ implies $U_{1}$ is in $U_{1}^{1}$. But this is impossible because $U_{1}$ includes $A$ and is in $U^{-1}$, and we have assumed there is no such set which goes into itself. Let

$$
U_{2}^{1}=C\left(U_{1} \cap U_{1}^{1}\right)
$$

so that

$$
U_{2}=C\left(U_{1}^{-1} \cap U_{1}\right) .
$$

Then $U_{2}$ is not in $U$, because $U_{2}$ is in $U^{-3} \cap U^{-2} \cap U^{-1}$ and if it were also in $U$ then $U_{2}$ would also be in $U_{1}^{1}$ and in $U_{2}^{1}$ which is impossible because $U_{2}$ includes $A$ and is in $U^{-1}$. Continuing step by step let

$$
U_{k}^{1}=C\left(U_{k-1} \cap U_{k-1}^{1}\right)
$$

so that

$$
U_{k}=C\left(U_{k-1}^{-1} \cap U_{k-1}\right) .
$$

Then $U_{k}$ is not in $U$ and we see this as follows: we have

$$
U_{k} \subset U^{-(k+1)} \cap \cdots \cap U^{-1}
$$

and

$$
U_{k}^{1} \subset U^{-k} \cap \cdots \cap U^{-1} \cap U,
$$


so that if $U_{k}$ were in $U$ then $U_{k}$ would also be in $U_{k}^{1}$ because it will then be in $U_{1}, U_{1}{ }^{1}, U_{2}, U_{2}{ }^{1}$, and so on, which is impossible as before.

Now let

$$
K=\bar{U}_{1} \cap \bar{U}_{2} \cap \cdots .
$$

Since it is the intersection of a monotone decreasing sequence of connected compact sets including $A$, it follows that $K$ also has these properties. It is clear that $K$ is in $\overline{U^{-1}}$, and since

$$
K^{1}=\overline{U_{1}^{1}} \cap \overline{U_{2}^{1}} \cap \cdots
$$

it also follows that $T(K) \subset K$ because $U_{k}{ }^{1} \subset U_{k-1}$. Each of the sets $\bar{U}_{i}$ includes a point not in $U$ and consequently $K$ must do the same so that $K$ must contain points not in $A$. The proof of Theorem $\mathrm{I}$ is thus complete.

COROLlaRY 1. If $T$ is a measure preserving homeomorphism of $E_{n}$ onto itself with a fixed point $p$, then there exist arbitrarily small compact connected closed sets, which contain $p$, and which are carried into themselves by $T$, and which contain more than one point.

CoROLlaRy 2. If $T$ is a measure preserving homeomorphism of $E_{n}$ onto itself with a fixed point $p$, then there exist arbitrarily large compact connected closed sets which contain $p$ and which are carried into themselves by $T$.

The proof of Corollary 1 follows immediately from the proof of Theorem 1. We turn to the proof of Corollary 2. Let $A_{1}$ be any compact connected set which includes $p$ and which is carried into itself by $T$. From Theorem I we know that we can find a larger set with similar properties and the proof of the corollary follows by transfinite induction or any similar technique.

CoRollary 3. Let $T$ be a measure preserving homeomorphism of $E_{n}$ onto itself and let $A$ be a compact connected set such that $T(A) \subset A$. Further let $U$ be an open set, including $A$ and having compact closure. Then there exists a compact connected set $K$ such that $A \subset K \subset \overline{U^{-1}}, K$ is not in $U$, and $T(K) \subset K$.

For the proof we must keep in mind the proof of Theorem 1 which has the same hypothesis. Notice that in either Case I or Case II we can assert that there exists a compact connected set larger than $A$ which goes into itself and which is in $\overline{U^{-1}}$. If this set is not in $U$ we may stop. If it is in $U$ we may proceed by transfinite induction to reach the conclusion.

The first two lemmas show the existence of both small and large 
continua of the type under discussion, but the third contains a slightly sharper result useful in the next theorem.

If $T(t ; x)$ is a one-parameter group of homeomorphisms of $E_{n}$ onto itself, then a point $p$ fixed under every element of the group is called a stationary point or a singular point of the group. The function $T(t ; x)$ is always assumed to be simultaneously continuous in $t$ and $x$.

THEOREM 2. Let $T(t ; x)$ be a one-parameter group of homeomorphisms of $E_{n}$ into itself and let $A$ be a compact connected set having the property that for all positive values of $t, A$ is mapped into itself. Then there exists a larger set with the same properties.

Let $U$ be an open connected set which includes $A$ and has a compact closure. For each $n$ we may find a set $K_{n}$ which is not in $U$ but is in $\overline{U^{-1}}$ and such that $T\left(1 / n ; K_{n}\right) \in K_{n}$. Some subsequence of this set of compact connected sets approaches (in the sense of Hausdorff distance [1]) a compact connected set $K$ which is in $\overline{U^{-1}}$ but not in $U$. We show now that for all positive values of $t$ this set is carried into itself. Let $x$ be any point of $K$ and let $x_{n}$ in $K_{n}$ be a sequence of points approaching $x$. It is clear that for all positive $k$ and $n$

$$
T\left(k / n ; x_{n}\right) \in K_{n} \text {. }
$$

Letting $t$ be any positive number it will be sufficient to prove that

$$
T(t ; x) \in K \text {. }
$$

Let $k_{n}$ be the largest non-negative integer such that $k_{n} / n<t$, so that as $n$ approaches infinity $k_{n} / n$ approaches $t$. We know that

$$
T\left(k_{n} / n ; x_{n}\right) \in K_{n} .
$$

Since $x_{n}$ approaches $x, k_{n} / n$ approaches $t$, and $T$ is simultaneously continuous we may conclude that $T(t ; x)$ is in $K$ which therefore ends the proof.

It follows from these considerations that we could find sets similar to those described in Theorem 1 and its corollaries for the negative iterates simply by letting $T^{-1}$ be the transformation. There need not be such sets which are carried into themselves by both $T$ and $T^{-1}$ as we can see for instance in the second example cited in the introduction. We may also make analogous remarks about the case of a oneparameter group.

Remark. It is well known that any continuous transformation of a closed disc into itself has a fixed point. An orientation preserving homeomorphism of the interior of a unit disc does not necessarily have a fixed point but if this homeomorphism is measure preserving 
then it must have a fixed point as we can see from the Brouwer translation theorem. We see from the above that there must be compact continua which include the fixed point, go into themselves and which extend as near as we please to the boundary of the disc. This fact may be of use in studying measure preserving homeomorphisms of the interior of a disc onto itself.

\section{BIBLIOGRAPHY}

1. Hausdorff, Mengenlehre, 3d ed., Berlin, 1935, p. 145.

2. Kerékjártó, Sur les similitudes de l'espace, C. R. Acad. Sci. Paris vol. 198 (1934) pp. 1345-1347.

Smith College 